\section{Impacto do efeito calendário na morbimortalidade por causas externas em municípios turísticos no Estado de São Paulo, Brasil, no período de 2004 a 2014}

\author{
Impact of calendar effect on morbidity and \\ mortality from external causes in tourist towns in \\ the State of São Paulo, Brazil, from 2004 to 2014
}

Impacto del efecto calendario en la morbimortalidad por causas externas en municipios turísticos en el Estado de São Paulo, Brasil, durante el período de 2004 a 2014
Paula Corrêa da Silva 1

Luiz Antonio Bastos Camacho 1

Cleber Nascimento do Carmo 1

\title{
Resumo
}

O objetivo desta pesquisa é analisar a associação de variações temporais dos agravos e das mortes por causas externas com períodos de maior fluxo de visitantes em municípios turísticos do litoral de São Paulo, Brasil, no período de 2004 a 2014. Trata-se de um estudo ecológico, de séries temporais diárias e mensais, baseado nos dados do Sistema de Informações sobre Mortalidade (SIM) e Sistema de Informações Hospitalares do Sistema Único de Saúde (SIH/SUS) no período de 1o de janeiro de 2004 a 31 de dezembro de 2014, tendo como unidades de análise os quatro municípios do litoral norte do Estado de São Paulo. Modelos de regressão binomial negativa foram ajustados para o desfecho que representou o número de ocorrências de internações hospitalares elou óbitos por causas externas, tendo como preditores as variáveis de calendário (dias da semana, feriados, mês e ano de ocorrência). Os feriados de Ano Novo, Carnaval, Tiradentes e Proclamação da República destacaram-se com médias iguais ou superiores a 5 desfechos por dia. Dentre os dias da semana, a segunda-feira e o domingo apresentaram as maiores médias. Levando em consideração todos os preditores, verifica-se maior tendência de ocorrência dos desfechos na segunda-feira (OR = 1,11; IC95\%: 1,05-1,18), nos feriados de Ano Novo (OR = 1,44; IC95\%: 1,19-1,74) e Proclamação da República $(O R=1,49$; IC95\%: 1,13-1,94) e nos meses de janeiro $(O R=1,11$; IC95\%: 1,02-1,20) e fevereiro $(O R=1,13$; IC95\%: 1,04-1,23). A morbidade e a mortalidade por causas externas nos municípios estudados foram maiores no período de maior movimentação de turistas, subsidiando a estruturação do atendimento destas lesões e as ações de prevenção e de promoção de saúde direcionadas para este grupo de causas.

Causas Externas; Morbidade; Mortalidade; Estações do Ano; Turismo

\author{
Correspondência \\ P. C. Silva \\ Av. Anchieta 206, bloco A, apto. 704, Caraguatatuba, SP \\ 11660-010, Brasil. \\ paulabotani@gmail.com \\ 1 Escola Nacional de Saúde Pública Sergio Arouca, Fundação \\ Oswaldo Cruz, Rio de Janeiro, Brasil.
}




\section{Introdução}

A denominação "causas externas" inclui agravos à saúde que têm determinantes acidentais ou intencionais 1. Dentre essas causas, os homicídios e os acidentes de trânsito lideram os indicadores de mortalidade no país. As mortes, os traumas e as lesões causadas por acidentes de transporte e por violência levam a altos custos emocionais e sociais e à grande utilização dos serviços de segurança pública, de saúde e de segurança no trânsito 2 . Como estratégia de prevenção de acidentes de transporte, foi desenvolvida, em 19 de junho de 2008, a Lei no 11.705 conhecida popularmente como Lei Seca com o objetivo de punir severamente o consumo de bebida alcóolica por parte do condutor do veículo automotor 3 .

A sazonalidade turística é definida como o desequilíbrio temporal no fenômeno do turismo e pode ser expressa em termos de dimensões de elementos como número de visitantes, gastos efetuados por eles, tráfego em rodovias e outras formas de transporte, e atrações festivas ${ }^{4}$, sendo tal efeito visto de forma positiva ou negativa na vida dos residentes locais. O turismo é um dos maiores e dos mais rápidos setores econômicos do mundo e, à medida em que ele aumenta, também aumenta a morbimortalidade associada a viagens 5 .

Rosselló \& Saenz-de-Miera 6 demonstraram como o aumento do turismo nas Ilhas Baleares (Espanha) está diretamente ligado ao aumento das mortes por acidentes. E, em um estudo anterior, Bentley et al. 7 observaram uma alta taxa de lesões e morbidade entre os visitantes, atribuível a atividades recreativas e de aventura na Nova Zelândia.

Ademais, Matos et al. 8 documentaram a maior divulgação de pesquisas, em anos recentes, sobre as condições de viajantes/turistas e seus riscos específicos. A mídia escrita e falada, com base em dados da segurança pública e da justiça penal, tem mostrado que após feriados prolongados e na alta temporada, principalmente nos feriados da Páscoa, do Natal e do Ano Novo, bem como no verão, há um aumento de mortes por causas externas.

O entendimento sobre o tipo e a frequência dos problemas de saúde em viagens é importante para a redução da morbidade e para a informação política, porém, existem dados científicos limitados sobre os problemas encontrados e a sua gravidade 5 . Com relação aos óbitos durante viagens ao exterior, esses números não estão disponíveis porque os países recebem viajantes e, assim, não contam com registros de morte centralizados. Dentre os óbitos, destacam-se aqueles provocados por acidentes de trânsito em que viajantes se comportam de maneira diferente, realizando atividades mais arriscadas, como dirigir sem cintos de segurança ou capacetes e consumir álcool antes de conduzir o veículo, ou quando não estão familiarizados com as condições e as práticas de condução. Tais situações colocam os viajantes em maior risco de lesões, mesmo em comparação com os motoristas locais 5,9,10.

O estudo desse fenômeno justifica-se pelos dados apresentados sobre a morbidade e a mortalidade por causas externas, seguidos da formulação de políticas de controle e prevenção de acidentes e de violências e do planejamento dos serviços. Além disso, a escassez de investigações sobre as variações das taxas de óbito por causas externas em municípios brasileiros turísticos e litorâneos que apresentam maior fluxo de pessoas e meios de transporte em períodos específicos durante o ano corrobora a importância da pesquisa.

Diante disso, o objetivo do presente trabalho foi analisar a associação de variações temporais dos agravos e das mortes por causas externas com períodos de maior fluxo de visitantes em municípios turísticos do litoral norte do Estado de São Paulo, Brasil, no período de 2004 a 2014. Essa região foi escolhida para a realização do estudo por concentrar grande fluxo de pessoas e pela disponibilidade de dados públicos pouco explorados.

\section{Métodos}

Trata-se de um estudo ecológico, exploratório, de séries temporais diárias e mensais recuperáveis de sistemas de informação públicos, disponíveis para consulta, tendo como unidades de análise os quatro municípios do litoral norte do Estado de São Paulo.

A região foi escolhida para a realização deste trabalho por concentrar grande fluxo de pessoas e pela disponibilidade de dados públicos pouco explorados. A área é composta por quatro municípios 
limítrofes: Caraguatatuba, Ilha Bela, São Sebastião e Ubatuba, que estão a aproximadamente 170km, 200km, 190km e 220km da Região Metropolitana de São Paulo, respectivamente, e contam com ligações rodoviárias como as rodovias Ayrton Senna, BR 101 (Rio-Santos), Oswaldo Cruz e Tamoios, facilitando o deslocamento de grande número de pessoas para os balneários.

Os dados foram obtidos no Sistema de Informações sobre Mortalidade (SIM) e no Sistema de Informações Hospitalares do Sistema Único de Saúde (SIH/SUS) no período de 1o de janeiro de 2004 a 31 de dezembro de 2014. Seguindo a Classificação Internacional de Doenças, 10a revisão (CID-10), foram selecionados os dados sobre hospitalizações por causas externas (capítulo XX) e por lesões, envenenamento e algumas outras consequências de causas externas (capítulo XIX) e óbitos por causas externas 1 .

Na configuração do banco de dados para modelagem, no primeiro momento, realizou-se a agregação das bases SIM e SIH/SUS, identificando o número do óbito e o número da internação por data de desfecho. Na base SIH/SUS, verificou-se que algumas internações findaram em óbitos. Então, para evitar duplicidade na junção das bases, esses casos foram excluídos com o propósito de manter apenas os eventos de internação. Em seguida, foi criada uma nova base de dados com óbitos e internações pelo SUS, reunindo as bases do SIM e do SIH/SUS segundo a data.

As variáveis explicativas que foram utilizadas para analisar a morbidade e a mortalidade por causas externas foram:

(a) Dia da semana da ocorrência: segunda-feira, terça-feira, quarta-feira, quinta-feira, sexta-feira, sábado e domingo (identificados valendo-se da data registrada na base de dados e em consulta ao calendário dos anos do estudo);

(b) Feriados nacionais: Natal (dias 24 e 25 de dezembro), Ano Novo (dias 31 de dezembro e 1o de janeiro), Carnaval (sábado, domingo, segunda-feira, terça-feira e Quarta-Feira de Cinzas), Semana Santa (sextafeira, sábado e domingo), Tiradentes (dia 21 de abril), Dia do Trabalho (dia 1o de maio), Corpus Christi (a quinta-feira), Independência do Brasil (dia 7 de setembro), Padroeira do Brasil (dia 12 de outubro), Finados (dia 2 de novembro) e Proclamação da República (dia 15 de novembro);

(c) Feriadão: dias consecutivos de folga prolongada devido a feriados nacionais próximos do final de semana e feriados que foram prolongados com fins de semana, segundas e sextas-feiras;

(d) Feriado em São Paulo: Revolução Constitucionalista de 1932 (dia 9 de julho) e Consciência Negra (dia 20 de novembro);

(e) Feriadão em São Paulo: feriados prolongados em São Paulo por estarem próximos do fim de semana; (f) Sexo: masculino ou feminino;

(g) Faixa etária: até 9 anos de idade, de 10 a 19, de 20 a 39, de 40 a 59, 60 anos ou mais, idade ignorada ou desconhecida;

(h) Raça: branca, preta, parda, ignorada ou desconhecida e outros (indígena e amarela); e

(i) Variável dicotômica relacionada à Lei Seca: 0 (antes da Lei Seca para desfechos de 1o de janeiro de 2004 a 18 de junho de 2008) e 1 (após a Lei Seca para desfechos de 19 de junho de 2008 a 31 de dezembro de 2014).

É importante destacar que a quarta-feira foi a categoria de referência para as estimativas da razão de chance, sendo escolhida por ser um dia com menos atividades de lazer, comparado aos fins de semana, mesmo em períodos de férias. Todas as variáveis explicativas usadas na análise do desfecho de interesse foram obtidas por meio do microdados dos sistemas de informação do Departamento de Informática do SUS (DATASUS), com exceção das variáveis sexo, faixa etária e raça que foram buscadas no TABNET do DATASUS (http://datasus.gov.br, acessado em 07/Abr/2016).

Inicialmente, foi realizada a análise exploratória dos agravos e das mortes por causas externas. Em seguida, adotou-se estratégia de modelagem da série temporal dos dados, via modelos lineares generalizados (MLG). No processo de estimação dos modelos verificou-se que a distribuição de Poisson não seria a melhor opção a ser usada para a construção dos modelos por ser observada uma superdispersão nos dados, levando a uma avaliação errônea da significância dos parâmetros de regressão. Dessa forma, foi estimado o modelo binomial negativo por apresentar as estatísticas mais consistentes quanto aos pressupostos e, como estratégia de controle do efeito sazonal, foram adicionadas ao modelo as variáveis mês e ano, e construídas duas bases de dados, com e sem outliers, por meio das análises individual e múltipla. 
Estimados os coeficientes do modelo, calculou-se a medida de associação razão de chance, que foi obtida pela exponenciação dos coeficientes de regressão. Baseando-se nos coeficientes estimados realizou-se uma análise da evolução temporal dos dados de morbimortalidade, investigando o papel do turismo e de eventos de calendário como feriados, fins de semana e férias escolares. Na avaliação e na seleção do modelo que melhor se ajustava, foi usado o teste da razão de verossimilhança (diferença entre as deviances) 11 .

A análise dos resíduos foi verificada por meio do uso do gráfico half normal plot (hnp) com envelope simulado. Também foi realizada a análise de resíduos padronizados usando-se as funções de autocorrelação (ACF) e funções de autocorrelação parciais (PACF), observado o critério de informação de Akaike para verificar o ajuste de cada modelo final 12,13. Todas as análises foram realizadas no programa estatístico R, na versão 3.3.3. (http://www.r-project.org).

A pesquisa foi baseada em dados secundários, sem identificação dos indivíduos e contemplou as recomendações da Resolução no 466/2012 do Conselho Nacional de Saúde. O trabalho foi aprovado em 11 de dezembro de 2016 pelo Comitê de Ética em Pesquisa da Escola Nacional de Saúde Pública Sergio Arouca, Fundação Oswaldo Cruz, segundo parecer no 1.860.000.

\section{Resultados}

A série histórica dos óbitos e das internações por causas externas segundo o local de ocorrência mostrou uma redução do número de desfechos ao longo dos meses quando se compara o início e o fim da série (Figura 1). É importante destacar que a série SIH/SUS apresentou período de queda até dezembro de 2009, seguido de discreto aumento e estabilização até dezembro de 2014.

Ainda foram observados, no decorrer do tempo da série analisada, picos de aumento (principalmente nos meses de janeiro e abril), com magnitude variando ao longo do período. Entre 2004 e 2008, as oscilações atingiram de 120 a 180 desfechos; entre 2009 e 2011, registraram de 100 a 140; e, por fim, a partir de 2012, as mesmas permaneceram entre 80 e 160 óbitos e internações.

A maioria dos óbitos envolveu o sexo masculino (85\%), na faixa etária de 20 a 39 anos (47\%), de raça branca (64\%) seguida da parda (25\%), sendo o perfil sociodemográfico dos óbitos e das internações semelhante nos quatro municípios.

Figura 1

Série histórica de óbitos e internações por causas externas segundo o local de ocorrência no litoral norte de São Paulo, Brasil, $2004-2014$.

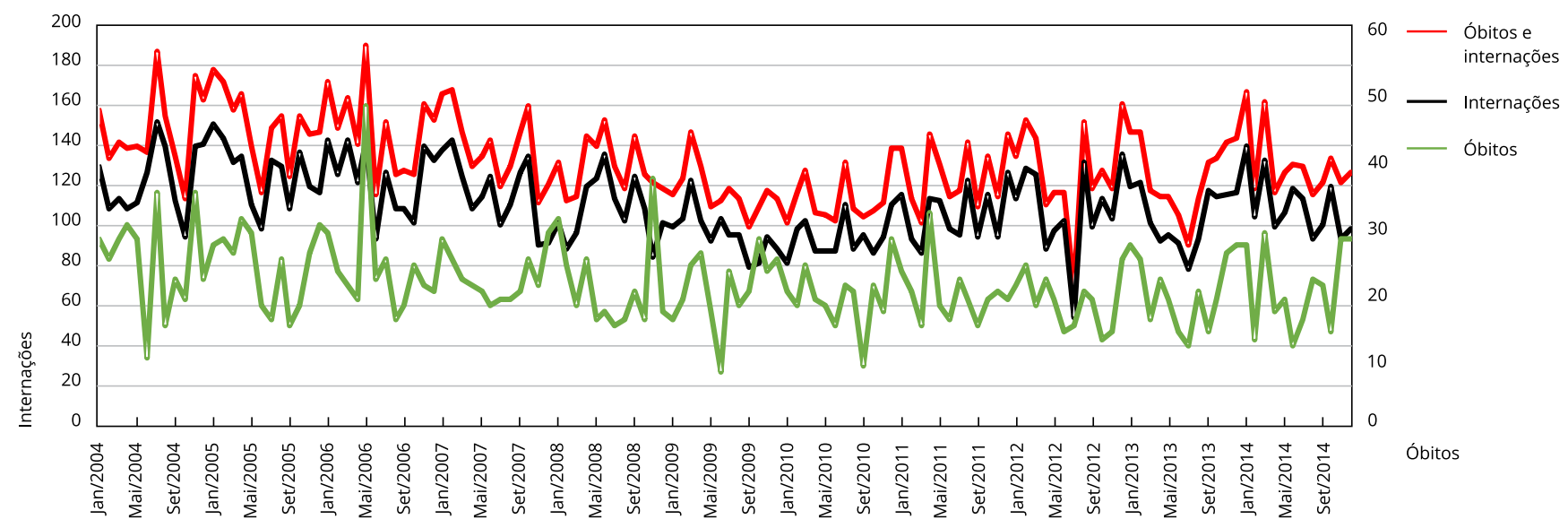


Nas internações por causas externas, foi observado maior envolvimento do sexo masculino (70\%), na faixa etária de 40 a 59 anos (31\%) e na raça branca (56\%). Na análise em conjunto dos óbitos e das internações por causas externas, observa-se a maior ocorrência no sexo masculino, nas faixas etárias de 20 a 39 anos (em torno de $30 \%$ ) e de 40 a 59 anos (em torno de 30\%), e na raça branca (em torno de 50\%), com exceção de Ilhabela, cuja predominância foi raça ignorada (83\%).

$\mathrm{Na}$ análise exploratória das variáveis explicativas realizada para o conjunto de dados de óbitos por causas externas, nota-se, nas variáveis relacionadas ao dia da semana, que as maiores médias e medianas de desfechos foram verificadas no sábado e no domingo (Tabela 1). No domingo, nota-se também maior desvio padrão, indicando uma maior variabilidade dos desfechos para essa variável ao longo do período analisado.

Para as demais variáveis (feriados ou período de festas), a maior média de óbitos foi verificada para a variável Ano Novo (média de 1,5 óbito e desvio padrão de 1,2 por dia, Tabela 1). Proclamação da República também apresentou média e mediana mais elevadas do que as demais datas festivas. Outras variáveis explicativas igualmente apresentaram médias e medianas substancialmente superiores às dos dias da semana sem feriados ou festividades, mesmo que possam ter sido influenciadas pelos períodos de férias.

$\mathrm{Na}$ análise das estatísticas descritivas das variáveis para o conjunto de dados de internações por causas externas, nota-se que, das variáveis relacionadas ao dia da semana, ao contrário dos óbitos, as maiores média e mediana foram apuradas na segunda-feira (Tabela 1). Nos outros dias da semana, as médias de internações foram semelhantes. Nas demais variáveis, as maiores média e mediana de internações foram apuradas nos feriados de Ano Novo e da Proclamação da República.

\section{Tabela 1}

Óbitos e internações por causas externas. Municípios do litoral norte de São Paulo, Brasil, 2004-2014.

\begin{tabular}{|c|c|c|c|c|c|c|c|c|c|c|}
\hline \multirow[t]{2}{*}{ Variável } & \multirow[t]{2}{*}{ Dias } & \multicolumn{3}{|c|}{ Óbitos } & \multicolumn{3}{|c|}{ Internações } & \multicolumn{3}{|c|}{ Internações e óbitos } \\
\hline & & Média & DP & Mediana & Média & DP & Mediana & Média & DP & Mediana \\
\hline Segunda-feira & 529 & 0,6 & 0,8 & 0 & 4,4 & 2,2 & 4 & 5,0 & 2,4 & 5 \\
\hline Terça-feira & 541 & 0,5 & 0,8 & 0 & 3,5 & 2,0 & 3 & 4,0 & 2,2 & 4 \\
\hline Quinta-feira & 547 & 0,6 & 0,8 & 0 & 3,9 & 2,1 & 4 & 4,5 & 2,3 & 4 \\
\hline Quarta-feira & 544 & 0,6 & 0,8 & 0 & 3,8 & 2,1 & 4 & 4,4 & 2,3 & 4 \\
\hline Sexta-feira & 520 & 0,6 & 0,8 & 0 & 3,2 & 2,0 & 3 & 3,8 & 2,2 & 3 \\
\hline Sábado & 463 & 0,9 & 1,1 & 1 & 3,1 & 1,9 & 3 & 3,9 & 2,2 & 4 \\
\hline Domingo & 463 & 1,0 & 1,4 & 1 & 3,5 & 2,0 & 3 & 4,5 & 2,7 & 4 \\
\hline Ano Novo & 22 & 1,5 & 1,2 & 1 & 5,3 & 2,5 & 6 & 6,8 & 3,2 & 7 \\
\hline Carnaval & 55 & 1,1 & 1,1 & 1 & 4,4 & 2,3 & 5 & 5,5 & 2,3 & 5 \\
\hline Semana Santa & 33 & 0,8 & 1,0 & 0 & 3,4 & 2,1 & 3 & 4,1 & 2,2 & 4 \\
\hline Tiradentes & 11 & 1,2 & 1,0 & 1 & 3,8 & 2,0 & 3 & 5,0 & 2,9 & 5 \\
\hline Dia do trabalho & 11 & 0,6 & 0,7 & 1 & 3,5 & 2,0 & 4 & 4,1 & 2,1 & 5 \\
\hline Corpus Christi & 11 & 0,7 & 0,9 & 1 & 2,9 & 2,4 & 2 & 3,6 & 2,5 & 3 \\
\hline Independência do Brasil & 11 & 0,8 & 0,8 & 1 & 3,6 & 2,2 & 4 & 4,5 & 2,7 & 5 \\
\hline Padroeira do Brasil & 11 & 0,8 & 1,1 & 0 & 3,5 & 2,1 & 3 & 4,4 & 2,6 & 4 \\
\hline Finados & 11 & 0,5 & 0,5 & 0 & 2,5 & 1,5 & 2 & 2,9 & 1,6 & 2 \\
\hline Proclamação da República & 11 & 1,4 & 1,0 & 2 & 5,3 & 4,4 & 5 & 6,6 & 4,9 & 7 \\
\hline Natal & 22 & 0,8 & 0,9 & 1 & 3,0 & 1,8 & 3 & 3,8 & 2,2 & 4 \\
\hline Feriadão & 297 & 0,9 & 1,0 & 1 & 3,5 & 2,2 & 3 & 4,4 & 2,5 & 4 \\
\hline Feriado em São Paulo & 15 & 1,3 & 1,2 & 1 & 2,7 & 2,0 & 3 & 3,9 & 2,2 & 4 \\
\hline Feriadão em São Paulo & 59 & 1,0 & 1,2 & 1 & 3,5 & 2,1 & 3 & 4,5 & 2,0 & 4 \\
\hline
\end{tabular}

DP: desvio padrão. 
E, por fim, a análise descritiva dos dados agregados dos óbitos e das internações por causas externas (Tabela 1) apresenta como destaque as variáveis Ano Novo, Carnaval, Tiradentes e Proclamação da República, todas com médias e medianas iguais ou superiores a 5 desfechos de óbitos ou de internações por dia. No caso do dia da semana, tem-se como destaque, com maiores médias e medianas, a segundafeira e o domingo, coerentes com os resultados das análises dos conjuntos de dados discutidos separadamente para óbitos e internações.

O impacto das variáveis explicativas propostas no número de óbitos e de internações por causas externas foi ajustado em modelo linear generalizado binomial negativo (Tabela 2). É importante destacar que, para o controle do efeito sazonal, foram adicionadas ao modelo variáveis dicotômicas referentes ao mês (mês de referência: agosto), como também variáveis dicotômicas associadas ao ano (ano de referência: 2004).

Tabela 2

Modelo final dos óbitos e internações por causas externas. Municípios do litoral norte de São Paulo, Brasil, 2004-2014.

\begin{tabular}{|c|c|c|c|c|c|c|}
\hline \multirow[t]{3}{*}{ Variável } & \multicolumn{6}{|c|}{ Análise múltipla } \\
\hline & \multicolumn{2}{|c|}{ SIM } & \multicolumn{2}{|c|}{ SIH/SUS } & \multicolumn{2}{|c|}{ SIM+SIH/SUS } \\
\hline & RC & IC95\% & RC & IC95\% & RC & IC95\% \\
\hline Constante & 0,60 & $0,48-0,74$ & 4,28 & $3,91-4,68$ & 4,87 & $4,48-5,3$ \\
\hline \multicolumn{7}{|l|}{ Feriados } \\
\hline Ano Novo & 2,02 & $1,32-3,03$ & 1,33 & $1,08-1,64$ & 1,44 & $1,19-1,74$ \\
\hline Carnaval & 1,85 & $1,33-2,54$ & 1,03 & $0,88-1,19$ & 1,13 & $0,98-1,30$ \\
\hline Semana Santa & 0,78 & $0,48-1,23$ & 0,95 & $0,76-1,18$ & 0,92 & $0,75-1,12$ \\
\hline Tiradentes & 1,51 & $0,77-2,77$ & 1,03 & $0,73-1,42$ & 1,11 & $0,82-1,49$ \\
\hline Trabalho & 0,82 & $0,33-1,73$ & 0,96 & $0,67-1,35$ & 0,94 & $0,67-1,28$ \\
\hline Corpus Christi & 1,05 & $0,45-2,17$ & 0,84 & $0,56-1,20$ & 0,86 & $0,60-1,20$ \\
\hline Independência & 1,29 & $0,61-2,48$ & 1,06 & $0,77-1,43$ & 1,09 & $0,81-1,45$ \\
\hline Aparecida & 1,06 & $0,48-2,08$ & 0,98 & $0,70-1,32$ & 0,99 & $0,73-1,32$ \\
\hline Finados & 0,54 & $0,19-1,26$ & 0,68 & $0,45-1,00$ & 0,65 & $0,44-0,94$ \\
\hline República & 1,58 & $0,84-2,84$ & 1,47 & $1,09-1,96$ & 1,49 & $1,13-1,94$ \\
\hline Natal & 0,95 & $0,54-1,60$ & 0,81 & $0,62-1,04$ & 0,83 & $0,65-1,05$ \\
\hline Feriadão & 1,50 & $1,22-1,84$ & 0,91 & $0,83-1,00$ & 0,99 & $0,90-1,08$ \\
\hline Feriado em São Paulo & 1,39 & $0,80-2,29$ & 0,75 & $0,53-1,03$ & 0,89 & $0,67-1,16$ \\
\hline Feriadão em São Paulo & 1,09 & $0,77-1,53$ & 1,00 & $0,84-1,18$ & 1,01 & $0,87-1,19$ \\
\hline Dummy Lei Seca & 1,02 & $0,77-1,35$ & 1,03 & $0,91-1,17$ & 1,03 & $0,92-1,15$ \\
\hline \multicolumn{7}{|l|}{ Dias da semana } \\
\hline Segunda-feira & 1,06 & $0,90-1,25$ & 1,12 & $1,05-1,19$ & 1,11 & $1,05-1,18$ \\
\hline Terça-feira & 0,94 & $0,79-1,11$ & 0,89 & $0,84-0,95$ & 0,90 & $0,85-0,96$ \\
\hline Quarta-feira & 1,04 & $0,88-1,22$ & 0,97 & $0,91-1,03$ & 0,98 & $0,92-1,04$ \\
\hline Quinta-feira & - & - & - & - & - & - \\
\hline Sexta-feira & 1,00 & $0,84-1,18$ & 0,82 & $0,77-0,88$ & 0,84 & $0,79-0,90$ \\
\hline Sábado & 1,50 & $1,29-1,76$ & 0,79 & $0,73-0,84$ & 0,88 & $0,82-0,94$ \\
\hline Domingo & 1,80 & $1,55-2,10$ & 0,90 & $0,84-0,96$ & 1,01 & $0,95-1,08$ \\
\hline \multicolumn{7}{|l|}{ Estatísticas } \\
\hline$\chi^{2}$ (valor de $p$ ) & \multicolumn{2}{|c|}{0,018} & \multicolumn{2}{|c|}{0,319} & \multicolumn{2}{|c|}{0,172} \\
\hline Durbin-Watson (valor de p) & \multicolumn{2}{|c|}{0,028} & \multicolumn{2}{|c|}{0,220} & \multicolumn{2}{|c|}{0,023} \\
\hline Deviance nulo & \multicolumn{2}{|c|}{4302,9} & \multicolumn{2}{|c|}{4676,0} & \multicolumn{2}{|c|}{4562,1} \\
\hline Deviance residual & \multicolumn{2}{|c|}{4068,0} & \multicolumn{2}{|c|}{4358,1} & \multicolumn{2}{|c|}{4249,3} \\
\hline
\end{tabular}

(continua) 
Tabela 2 (continuação)

\begin{tabular}{|c|c|c|c|c|c|c|}
\hline \multirow[t]{3}{*}{ Variável } & \multicolumn{6}{|c|}{ Análise múltipla } \\
\hline & \multicolumn{2}{|c|}{ SIM } & \multicolumn{2}{|c|}{ SIH/SUS } & \multicolumn{2}{|c|}{ SIM+SIH/SUS } \\
\hline & $\mathbf{R C}$ & IC95\% & RC & IC95\% & RC & IC95\% \\
\hline \multicolumn{7}{|l|}{ Mês } \\
\hline Janeiro & 1,25 & $1,02-1,52$ & 1,09 & $1,00-1,18$ & 1,11 & $1,02-1,20$ \\
\hline Fevereiro & 1,19 & $0,96-1,46$ & 1,12 & $1,03-1,23$ & 1,13 & $1,04-1,23$ \\
\hline Março & 1,13 & $0,93-1,39$ & 1,05 & $0,97-1,15$ & 1,06 & $0,98-1,15$ \\
\hline Abril & 1,29 & $1,06-1,58$ & 1,00 & $0,91-1,09$ & 1,04 & $0,96-1,13$ \\
\hline Maio & 1,18 & $0,97-1,45$ & 0,98 & $0,90-1,07$ & 1,01 & $0,93-1,10$ \\
\hline Junho & 0,79 & $0,64-0,99$ & 1,00 & $0,92-1,09$ & 0,97 & $0,90-1,05$ \\
\hline Julho & 1,02 & $0,83-1,25$ & 0,97 & $0,89-1,05$ & 0,97 & $0,90-1,06$ \\
\hline Agosto & - & - & - & - & - & - \\
\hline Setembro & 0,94 & $0,76-1,16$ & 0,99 & $0,90-1,08$ & 0,98 & $0,90-1,06$ \\
\hline Outubro & 1,02 & $0,83-1,25$ & 0,99 & $0,91-1,08$ & 0,99 & $0,92-1,08$ \\
\hline Novembro & 1,27 & $1,04-1,56$ & 0,99 & $0,90-1,08$ & 1,03 & $0,95-1,12$ \\
\hline Dezembro & 1,25 & $1,03-1,53$ & 1,03 & $0,95-1,12$ & 1,06 & $0,98-1,15$ \\
\hline \multicolumn{7}{|l|}{ Ano } \\
\hline 2004 & - & - & - & - & - & - \\
\hline 2005 & 0,98 & $0,82-1,17$ & 1,03 & $0,95-1,11$ & 1,02 & $0,95-1,09$ \\
\hline 2006 & 0,97 & $0,81-1,15$ & 1,01 & $0,93-1,09$ & 1,00 & $0,93-1,07$ \\
\hline 2007 & 0,90 & $0,75-1,07$ & 0,95 & $0,88-1,03$ & 0,94 & $0,88-1,01$ \\
\hline 2008 & 0,85 & $0,67-1,06$ & 0,86 & $0,78-0,95$ & 0,86 & $0,78-0,94$ \\
\hline 2009 & 0,82 & $0,59-1,14$ & 0,76 & $0,66-0,88$ & 0,77 & $0,67-0,88$ \\
\hline 2010 & 0,76 & $0,54-1,07$ & 0,74 & $0,64-0,86$ & 0,74 & $0,65-0,85$ \\
\hline 2011 & 0,80 & $0,57-1,12$ & 0,83 & $0,72-0,96$ & 0,83 & $0,72-0,95$ \\
\hline 2012 & 0,74 & $0,53-1,04$ & 0,85 & $0,73-0,98$ & 0,83 & $0,72-0,95$ \\
\hline 2013 & 0,81 & $0,58-1,13$ & 0,82 & $0,71-0,95$ & 0,82 & $0,72-0,94$ \\
\hline 2014 & 0,83 & $0,59-1,15$ & 0,87 & $0,75-1,00$ & 0,86 & $0,75-0,98$ \\
\hline
\end{tabular}

IC95\%: intervalo de 95\% de confiança; RC: razão de chance; SIH/SUS: Sistema de Informações Hospitalares do Sistema Único de Saúde; SIM: Sistema de Informações sobre Mortalidade.

Após, foi realizada a análise das estimativas das razões de chances de desfechos (óbitos e internações) da base agregada, em que verificou-se significância estatística nas variáveis dia da semana - segundafeira (chances $11 \%$ maiores), terça-feira (10\% menores), sexta-feira (16\% menores) e sábado (chances $12 \%$ menores) - e feriados: Ano Novo (chances 44\% maiores), Finados (35\% menores) e Proclamação da República (chances 49\% maiores).

No que diz respeito à variável dicotômica inserida no modelo que buscou avaliar o impacto da Lei Seca no número de desfechos, não foi possível evidenciar estatisticamente, na análise múltipla, que a implantação da referida lei reduziu ou aumentou o número de óbitos e internações por causas externas. No entanto, na análise individual indica-se que, após a implantação da Lei Seca, a chance de óbitos por causas externas foi reduzida em 16\%. Do mesmo modo, o resultado de internações por causas externas indica $15 \%$ menos chances quando comparado àquele do período precedente à Lei Seca. $\mathrm{Na}$ base desagregada, a variável dicotômica associada à citada lei não foi significante na análise múltipla, mas significativa na análise individual.

No tocante às estimativas dos efeitos sazonais sobre os óbitos, as variáveis significantes foram as mesmas do modelo cuja variável dependente era número de internações por causas externas (SIH/SUS): janeiro (chances de desfecho 11\% maiores) e fevereiro (chances de desfecho 13\% maiores) e, no ano, as variáveis que representam o período de 2008 a 2014, em que se destaca o ano de 2010, com 26\% menos chances de desfechos quando comparado a 2004. A escolha do modelo final apresentado na Tabela 2 foi realizada mediante a análise de resíduos padronizados pelas funções de autocorrelação e autocorrelação parcial dos resíduos. 


\section{Discussão}

A pesquisa identificou a tendência de queda dos óbitos e das internações por causas externas no litoral norte do Estado de São Paulo. Observa-se que a morbidade e a mortalidade por causas externas nos municípios da região, no período de 2004 a 2014, foram maiores na alta temporada, considerando o período de festas de final de ano, como Ano Novo, férias ou recesso de final de ano nos meses de dezembro e de janeiro, e em outros meses, como junho e novembro, por terem feriados. O dia da semana também pode estar associado à movimentação turística, com o aumento das internações na segunda-feira, que é o primeiro dia após o fim de semana.

Nos municípios analisados, a frequência de óbitos por causas externas foi cerca de três vezes maior nos homens do que nas mulheres, e a de internações foi cerca de duas vezes maior. Tais resultados são semelhantes aos de pesquisa realizada por Costa et al. $14 \mathrm{em}$ Pelotas (Rio Grande do Sul), na qual a taxa de mortalidade por causas externas foi maior entre os homens do que entre as mulheres, sendo a maior taxa encontrada de 15 a 39 anos. Assim como em um estudo realizado em Diamantina (Minas Gerais), em que foi evidenciado maior risco de morte para homens (coeficiente médio $=71,5 / 100$ mil) 15 .

Com relação à idade, os resultados encontrados são semelhantes aos de outras pesquisas, em que foram observadas causas externas com maior gravidade, com óbito nos adultos jovens por experimentarem situações de risco e por abusarem de substâncias psicoativas 16,17.

A maior média de óbitos no sábado e no domingo, observada neste estudo, foi semelhante à encontrada na pesquisa de Bando 18. Provavelmente, isso se deve ao fato de serem dias de folga ou lazer, frequentemente associados ao uso de álcool e de drogas ilícitas, favorecendo maior letalidade.

$\mathrm{Na}$ análise das internações, as maiores médias foram encontradas na segunda-feira e, na análise do conjunto de internações e de óbitos, a segunda-feira e o domingo apresentaram as maiores médias. A frequência de internações nas segundas-feiras pode ser inflada pelos eventos no fim de semana, em que indivíduos são hospitalizados após permanecerem em observação nos serviços de pronto atendimento até o surgimento de vaga para internação.

Em relação à variável feriado, observa-se que os períodos comemorativos contribuíram para o aumento dos óbitos e das internações por causas externas. Esse fato corrobora as notícias publicadas em jornais escritos, on-line e falados sobre o aumento de acidentes de trânsito e de violência nos feriados. Em fevereiro de 2018, foram registrados, pela Polícia Rodoviária Federal (PRF), 249 acidentes graves e 87 óbitos nas rodovias federais durante o feriado de Carnaval. Outro evento ocorrido e noticiado em dezembro de 2016 foi o aumento de 54\% nos roubos e nos furtos em Caraguatatuba (São Paulo), assim como em outros municípios do litoral de São Paulo, sendo proposto pelo Governo do Estado, a partir de 19 de dezembro de 2016, uma operação verão com a oferta de mais policiamento nas praias paulistas 19,20.

Além desses eventos noticiados, reforça os achados citados anteriormente o estudo realizado em Pernambuco que identificou a ocorrência da influência sazonal nos acidentes com ciclistas no período de janeiro 2012 a dezembro de 2014. Na referida pesquisa observou-se que no verão (meses de dezembro, janeiro e fevereiro) e na primavera (meses de setembro, outubro e novembro) foi registrada a maioria dos acidentes 21. Outros autores também relatam a importância da associação entre a ocorrência de acidentes e as estações do ano, visto que o número de viagens pode variar de acordo com as condições do tempo (temperatura, umidade e clima) 22,23.

A literatura sobre a influência da sazonalidade turística na ocorrência da morbimortalidade por causas externas é escassa, evidenciando apenas isoladamente o tema turismo ou este associado a doenças infecciosas ou somente a causas externas.

$\mathrm{O}$ aumento dos desfechos está, eventualmente, relacionado a feriados como Independência do Brasil, Padroeira do Brasil, Finados e Proclamação da República (abrangendo os meses de setembro a novembro) e no Natal, Ano Novo, Carnaval e na Páscoa compreendidos entre os meses de dezembro e março, período considerado como de alta temporada nesses municípios turísticos e litorâneos em razão do verão e das férias escolares, aumentando a população total da região devido aos veranistas e aos turistas provenientes de outros municípios do Estado de São Paulo. O feriado de Tiradentes, no período estudado, não ocorreu em nenhum dos meses citados. Dessa forma, intui-se que o número de desfechos pode ter aumentado naquela data por se tratar de feriado prolongado em alguns dos anos analisados, como em 2005, 2006, 2008, 2009, 2011 e 2014 ou, ainda, por estar fora da alta temporada, o contraste com os dias da semana pode ter ficado mais acentuado. 
Com relação à variável dicotômica Lei Seca, apenas na análise múltipla, das três propostas de modelo estudadas, esta variável não apresentou relevância estatística na ocorrência dos desfechos por causas externas. Porém, na análise individual, após a Lei Seca, as chances dos desfechos são reduzidas quando comparadas ao período precedente a essa proposta. Em vista disso, a variável Lei Seca pode ter sido um possível modificador do efeito das variáveis que se mostraram relevantes na ocorrência dos desfechos do presente estudo, podendo ter afetado a análise dos resultados. Parte do efeito aparente da citada lei resultava do confundimento das demais variáveis. De acordo com uma pesquisa sobre regressão segmentada 24 , só haveria sentido aplicá-la no presente modelo se houvesse redução ou aumento na série de desfecho após a Lei Seca, mas, aparentemente, não foi o caso.

Após a Lei Seca, segundo Mello-Jorge \& Adura ${ }^{25}$, as medidas legais contra o uso de bebidas alcoólicas associado à direção veicular, evidenciaram a redução do número de acidentes de trânsito e do impacto nos óbitos e na morbidade das vítimas resgatadas pelo Serviço de Atendimento Móvel de Urgência (SAMU) no Brasil.

Algumas limitações do estudo devem ser consideradas na análise dos resultados apresentados. As internações hospitalares podem não evidenciar toda a morbidade por causas externas, dado que uma parte das vítimas não chega a ser atendida nos serviços de saúde locais de urgência e emergência ou é liberada logo após o atendimento. Hospitalizações e óbitos de turistas tenderiam a ocorrer nos municípios de residência, atenuando a sazonalidade. Outra limitação é que as internações particulares e atendidas por planos de saúde não estão incluídas na base de dados explorada. Se entre os turistas a proporção de usuários de planos de saúde for maior do que na população residente nos municípios, a frequência de óbitos e hospitalizações terá sido subestimada.

É possível que o impacto do fluxo de turistas tenha sido subestimado pelo sub-registro dos eventos de interesse para o estudo. Além disso, a própria insuficiência de recursos de saúde postulada nesta pesquisa pode fazer com que as hospitalizações e os óbitos por agravos ocorridos nesses municípios ocorram fora deles. Sendo assim, o impacto do fluxo turístico na morbimortalidade pode ter sido subestimado.

Destaca-se, além de tudo, que este estudo é original e o tema é incomum na área acadêmica. Foram aplicadas técnicas analíticas para séries temporais que permitiram alcançar novos insights com base nos dados apresentados, possibilitando a exposição dos resultados deste trabalho aos gestores dos municípios estudados e a discussão da incorporação de análises mais simples, como a apresentada na Figura 1, à vigilância dos municípios.

Esta pesquisa, apesar das limitações referidas, é de extrema relevância para a análise de municípios com significativa movimentação turística e impactos sobre a saúde ainda pouco conhecidos. Considerando a importância das atividades turísticas para a economia dessas pequenas localidades, há necessidade de melhorar os recursos utilizados para o atendimento à saúde da população fixa e flutuante, com investimentos e planejamento dos serviços de segurança pública, como o aumento do policiamento e da fiscalização nas estradas, entre outras medidas preventivas.

Além disso, é fundamental o aumento do número de leitos hospitalares, de ambulâncias, de recursos humanos qualificados e de especialidades médicas, de modo a tornar esses municípios aptos a atender a demanda extra por serviços de saúde locais nos períodos de férias e feriados. 


\section{Colaboradores}

P. C. Silva contribuiu na concepção e planejamento do estudo, análise e interpretação dos resultados, coleta e análise dos dados, elaboração do texto, revisão do manuscrito e aprovação da versão final. L. A. B. Camacho e C. N. Carmo contribuíram na concepção e planejamento do estudo, análise e interpretação dos resultados, revisão do manuscrito e aprovação da versão final.

\section{Informações adicionais}

ORCID: Paula Correa da Silva (0000-0002-29855470); Luiz Antonio Bastos Camacho (0000-00034656-1914); Cleber Nascimento do Carmo (00000003-4165-2198).

\section{Referências}

1. World Health Organization. ICD-11 for mortality and morbidity statistics. https://icd. who.int/browse11/1-m/en (acessado em 06/ Mai/2018).

2. Bastos MJRP, Pereoa JA, Smarzaro DC, Costa EF, Bossanel RCL, Oliosa DMS, et al. Análise ecológica dos acidentes e da violência letal em Vitória - ES. Rev Saúde Pública 2009; 43:12332.

3. Brasil. Lei no 11.705, de 19 de junho de 2008. Altera a Lei no 9.503, de 23 de setembro de 1997, que 'institui o Código de Trânsito Brasileiro', e a Lei no 9.294, de 15 de julho de 1996, que dispõe sobre as restrições ao uso e à propaganda de produtos fumígeros, bebidas alcoólicas, medicamentos, terapias e defensivos agrícolas, nos termos do $₫ 4$ o do art. 220 da Constituição Federal, para inibir o consumo de bebida alcoólica por condutor de veículo automotor, e dá outras providências. Diário Oficial da União 2008; 20 jun.

4. Baum T, Lundtorp S. Seasonality in tourism. Oxford: Pergamum; 2001.

5. Behrens RH, Carrol B. Travel trends and patterns of travel-associated morbidity. Infect Dis Clin North Am 2012; 26:791-802.

6. Rosselló J, Saenz-de-Miera O. Road accidents and tourism: the case of the Balearic Islands (Spain). Accid Anal Prev 2011; 43:675-83.

\section{Agradecimentos}

À Coordenação de Aperfeiçoamento de Pessoal de Nível Superior (CAPES).

7. Bentley T, Meyer D, Page S, Chalmers D. Recreational tourism injuries among visitors to New Zealand: an exploratory analysis using hospital discharge data. Tour Manag 2001; 22:373-81.

8. Matos V, Barcellos C, Camargo LOL. Vulnerabilidade e problemas de saúde em viagem: a visão do turista na Cidade do Rio de Janeiro. Ciênc Saúde Colet 2013; 18:85-97.

9. Hargarten SW, Baker TD, Guptill K. Overseas fatalities of United States citizen travelers: an analysis of deaths related to international travel. Ann Emerg Med 1991; 20:622-6.

10. Mac Pherson DW, Gushulak BD, Sandhu J. Death and international travel - the Canadian experience: 1996 to 2004. J Travel Med 2007; 14:77-84.

11. Cameron AC, Trivedi PK. Regression analysis of count data. New York: Cambridge University Press; 1998.

12. Wood SN. Generalized additive models: an introduction with R. Boca Raton/New York/ Abingdon: Chapman \& Hall/CRC Press; 2006.

13. Faraway JJ. Extending the linear model with R. Boca Raton/New York/Abingdon: Chapman \& Hall/CRC; 2006. (Texts in Statistical Science Series). 
14. Costa JSD, Giraldi MC, Carret MLV, Ferreira AMTB, Strauch ES, Moraes M. Evolução da mortalidade por causas externas no Município de Pelotas e no Estado do Rio Grande do Sul, Brasil, 1996-2009. Epidemiol Serv Saúde 2013; 22:215-24.

15. Corassa RB, Falci DM, Gontijo CF, Machado GVC, Alves PAB. Evolução da mortalidade por causas externas em Diamantina - MG, 2001 a 2012. Cad Saúde Colet (Rio J.) 2017; 25:30214.

16. Organização Pan-Americana da Saúde. Uso de drogas e segurança no trânsito. http://iris.paho.org/xmlui/bitstream/hand le/123456789/34979/OPASBRA18012-por. pdf? sequence $=1 \&$ isAllowed $=y$ (acessado em 06/Mai/2018).

17. Secretaria de Vigilância em Saúde, Ministério da Saúde. Painel temático de indicadores do SUS: prevenção de violências e cultura da paz III. Brasília: Organização Pan-Americana da Saúde; 2008.

18. Bando DH. Sazonalidade, efemérides e a mortalidade por doença coronariana, AVC, insuficiência cardíaca, acidente de transporte, suicídio e homicídio na Cidade de São Paulo, 1996 a 2009 [Tese de Doutorado]. São Paulo; Faculdade de Medicina, Universidade de São Paulo; 2012.

19. Verdélio A. PRF registra 249 acidentes graves no feriado de carnaval com 87 mortos. Portal do Trânsito e Mobilidade 2018; 14 fev. http:// portaldotransito.com.br/noticias/prf-regis tra-249-acidentes-graves-no-feriado-de-car naval-com-87-mortos/.
20. Machado L. Litoral paulista enfrenta disparada de roubos; alta supera capital e Estado. Folha de S.Paulo 2016; 19 mai. http://www1.folha. uol.com.br/cotidiano/2016/12/1838233-li toral-paulista-enfrenta-disparada-de-roubosalta-supera-capital-e-estado.shtml.

21. Nóbrega KBV. Padrão sazonal, espacial, fatores associados e o itinerário dos acidentes com ciclistas em Pernambuco, 2012 a 2014 [Dissertação de Mestrado]. Recife: Centro de Pesquisas Aggeu Magalhães, Fundação Oswaldo Cruz; 2016.

22. Zhang XZ, Pang Y, Cui M, Stallones L, Xiang $\mathrm{H}$. Forecasting mortality of road traffic injuries in China using seasonal autoregressive integrated moving averege model. Ann Epidemiol 2015; 25:101-6.

23. Craig J, Hinds JD, Kealeu DWC, Heyes GJ. The burden of motorcycle trauma and seasonal change at a regional trauma centre. Ulster Med J 2014; 83:55-6.

24. Wagner AK, Soumerai SB, Zhang F, Ross-Degnan D. Segmented regression analysis of interrupted time series studies in medication use research. J Clin Pharm Ther 2002; 27:299-309.

25. Mello-Jorge MHP, Adura FE. Álcool e direção veicular. Revista USP 2013; 96:23-36. 


\section{Abstract}

This study aims to analyze the association between temporal variations in injuries and deaths from external causes and periods of greater flow of visitors in tourist municipalities (counties) on the coastline of the state of São Paulo, Brazil, from 2004 to 2014. This is an ecological study of daily and monthly time series based on data from the Brazilian Mortality Information System (SIM) and Brazilian Hospital Information System of the Brazilian Unified National Health System (SIH/ SUS) from January 1, 2004, to December 31, 2014, using as the analytical units the four municipalities on the northern coast of the State of São Paulo. Negative binomial regression models were adjusted for the outcome that represented the number of hospital admissions and/or deaths from external causes, with calendar variables as predictors (days of the week, holidays, month, and year of occurrence). The Brazilian holidays New Year's Day, Carnival, Tiradentes, and Proclamation of the Republic stood out with mean numbers greater than or equal to 5 outcomes per day. Among the days of the week, Monday and Sunday had the highest mean numbers. Considering all the predictors, there was a higher tendency to the occurrence of outcomes on Monday (OR $=1.11$; 95\% CI: 1.05-1.18), New Year's Day (OR = 1.44; 95\%CI: 1.19-1.74), and Proclamation of the Republic Day $(O R=1.49 ; 95 \% C I: 1.13-1.94)$ and in the months of January $(O R=1.11 ; 95 \% C I: 1.02-1.20)$ and February $(O R=1.13$; 95\%CI: 1.04-1.23). Morbidity and mortality from external causes in these tourist towns were higher in the period with the greatest flow of tourists, emphasizing the need to organize care for these injuries, alongside measures for prevention and health promotion targeted to this group of causes.

External Causes; Morbidity; Mortality; Seasons; Tourism

\section{Resumen}

El objetivo de esta investigación es analizar la asociación de variaciones temporales en enfermedades y muertes por causas externas, durante periodos de mayor flujo de visitantes en municipios turísticos del litoral de São Paulo, Brasil, durante el periodo de 2004 a 2014. Se trata de un estudio ecológico, de series temporales diarias y mensuales, basado en los datos del Sistema de Información sobre Mortalidad (SIM) y Sistema de Informaciones Hospitalarias del Sistema Único de Salud (SIH/SUS), durante el período del 1o de enero de 2004 al 31 de diciembre de 2014, teniendo como unidades de análisis los cuatro municipios del litoral norte del Estado de São Paulo. Modelos de regresión binomial negativa se ajustaron al resultado que representó el número de ocurrencias de internamientos hospitalarios $y / u$ óbitos por causas externas, teniendo como predictores variables de calendario (días de la semana, festivos, mes y año de ocurrencia). Los festivos de Año Nuevo, Carnaval, Tiradentes y Proclamación de la República se destacaron con medias iguales o superiores a 5 resultados por día. Entre los días de la semana, el lunes y el domingo presentaron las mayores medias. Teniendo en consideración todos los predictores, se verifica una mayor tendencia de ocurrencia de los resultados: los lunes $(O R=1,11$; IC95\%: 1,05-1,18), festivo de Año Nuevo $(O R=1,44$; IC95\%: 1, 19-1,74) y Proclamación de la República $(\mathrm{OR}=1,49$; IC95\%: 1,13-1,94) $y$, en los meses de enero $(O R=1,11$; IC95\%: 1,02-1,20) y de febrero $(\mathrm{OR}=1,13$; IC95\%: 1,04-1,23). La morbilidad y mortalidad por causas externas en los municipios estudiados fueron mayores en el período de mayor movimiento de turistas, apoyando la organización de la atención a esas lesiones, así como acciones de prevención y promoción de salud dirigidas hacia este grupo de causas.

Causas Externas; Morbilidad; Mortalidad; Estaciones del Año; Tourismo
Recebido em 20/Set/2019

Versão final reapresentada em 30/Abr/2020

Aprovado em 19/Jun/2020 
Silva PC, Camacho LAB, Carmo CN. Impacto do efeito calendário na morbimortalidade por causas externas em municípios turísticos no Estado de São Paulo, Brasil, no período de 2004 a 2014. Cad Saúde Pública 2021; 37(1):e00174019.

doi: 10.1590/0102-311XER174019

Onde se lê:

Paula Correia da Silva 1

Luiz Antonio Bastos Camacho 1

Cleber Nascimento do Carmo 1

Leia-se:

Paula Corrêa da Silva 1

Luiz Antonio Bastos Camacho ${ }^{1}$

Cleber Nascimento do Carmo 1 